\title{
RADIATIVELY DRIVEN WINDS OF OB STARS
}

\author{
MICHIHIRO R. SHIMADA, MASAKI ITO*, RYUKO HIRATA \\ Department of Astronomy, Kyoto University, Sakyo-ku, Kyoto, 606-01, Japan \\ and \\ TOSHIHIRO HORAGUCHI \\ National Science Museum, Ueno Park, Taito-ku, Tokyo 110, Japan
}

\begin{abstract}
We newly calculated the line radiative force with 520,000 atomic lines, which is twice as many as those of Abbott (1982), for OB supergiants. Our results are as follows. (1) The mass loss rates for $O$ stars with $T_{\text {eff }}=50,000 \mathrm{~K}$ are seven times as large as Abbott's (1982) because of contribution from Fe IV lines. (2) Contribution from many weak lines increases the mass loss rates and decreases the wind velocities of OB stars within a temperature range of $10,000 \mathrm{~K} \leq T_{\text {eff }} \leq 30,000 \mathrm{~K}$. This result is qualitatively in accordance with the results from the recent observations of $O$ stars. (3) The mass loss rates of OB stars depend on metallicity with $\dot{M} \sim Z$.
\end{abstract}

OB stars lose their mass through stellar winds. Line radiative force is thought to be the dominant mechanism for driving winds. Analytical formulation for line driven wind was developed by Castor, Abbott \& Klein (1975). Abbott (1982) calculated the line radiative force with a list of 250,000 atomic lines and investigated the properties of the force in detail. The numerical values of the line radiative force of Abbott (1982) have been widely used in the study of mass loss phenomena of OB stars so far.

We calculated the line radiative force with ATMLINE database, which is more complete database of opacity and excitation level of atomic lines. We took into account the first to sixth stages of ionization of the elements $\mathrm{H}-$ $\mathrm{Ni}$ and the first to forth stages of ionization of the elements $\mathrm{Cu}-\mathrm{U}$. Total number of absorption lines amounts to 520,000 , which is twice as many as those of Abbott (1982).

The line radiative force was calculated with Sobolev approximation, as in Castor, Abbott \& Klein (1975) and Abbott (1982). Following Pauldrach et al. (1986), the line radiative force per unit mass is expressed as

$$
\frac{\kappa_{\mathrm{e}} F}{c} f_{\text {rad }} F_{\text {corr. }}
$$

where $\kappa_{\mathrm{e}} F / c$ is the acceleration due to continuum radiation pressure and $F_{\text {corr. }}$ is a correction factor for a finite core angle. The force multiplier, $f_{\text {rad }}$, can be approximated by

$$
f_{\mathrm{rad}} \sim k t^{-\alpha} s^{\delta}
$$

* Deceased on 27 March, 1989 
as a function of $t$ and $s$, where the value $t$ and $s$ are defined as

$$
t=\kappa_{\mathrm{e}} \rho v_{\mathrm{th}}\left|\frac{d v}{d r}\right|^{-1}, s=\frac{\rho}{m_{\mathrm{H}} W} \times 0.85 \times 10^{-11} .
$$

All quantities have the same meaning as in Abbott (1982).

The force multiplier and the values $k, \alpha$ and $\delta$ were calculated for OB supergiants within the temperature range $10,000 \mathrm{~K} \leq T_{\text {eff }} \leq 50,000 \mathrm{~K}$ and within the metallicity range $0.002 \leq Z \leq 0.165$. The results for the case with $Z=0.02$ are listed in Table 1 and Table 2 .

We found that the line radiative force for the star with $T_{\text {eff }}=50,000 \mathrm{~K}$ is larger by $0.3-0.5$ dex than Abbott's (1982) because our new atomic line database includes much more highly ionized ions, such as Fe IV. Accordingly the mass loss rate for this star is seven times as large as Abbott's (1982).

Our new atomic line database also includes much more weak lines than before. Therefore, the force multiplier parameter $k$ becomes larger by a factor of 1.6-3.8 and $\alpha$ becomes smaller by 0.05 than Abbott's (1982) within a temperature range of $10,000 \mathrm{~K} \leq T_{\text {eff }} \leq 30,000 \mathrm{~K}$. Groenewegen et al. (1989) and Lamers \& Leitherer (1993) recently pointed out that the predicted terminal velocities for $\mathrm{O}$ stars are systematically larger than the observed values by about a factor of 1.4 , and that the predicted mass loss rates are systematically smaller than the observed values by about $-2.5 \mathrm{dex}$. Since our force multiplier parameters tend to increase mass loss rates and decrease wind velocities, it is expected to dissolve the discrepancies between predictions and observation. This result shall be refined by future works.

We found that the mass loss rates for metal deficient stars depend on metallicity roughly with $\dot{M} \sim Z$. This result is close to the result of Abbott (1982) rather than that of Kudritzki et al. (1987). We all have succeeded in interpretation of the wind velocities of Magellanic $\mathrm{O}$ stars but failed to interpret the observational mass loss rates of Magellanic $O$ stars. This is one of the most challenging problems of the stellar wind theory.

\section{References}

Abbott, D.C. 1982, Ap. J., 259, 282.

Castor, J., Abbott, D.C. and Klein, R. 1975, Ap. J., 195, 157.

Groenewegen, M.A.T., Lamers, H.J.G.L.M. and Pauldrach, A.W.A. 1989, A. Ap., 221, 78

Kudritzki, R.P., Pauldrach, A.W.A. and Puls, J. 1987, A. Ap., 173, 293.

Lamers, H.J.G.L.M and Leitherer, C. 1993, Ap. J., 412, 771.

Pauldrach, A.W.A., Puls, J. and Kudritzki, R.P. 1986, A. Ap., 164, 86. 
Table 1: The force multiplier for $\mathrm{OB}$ supergiants

\begin{tabular}{|rll|rrrrrrr|}
\hline \hline$T_{\text {eff }}$ & $\log g$ & \multirow{2}{*}{$s$} & \multicolumn{7}{|c|}{$\log f_{\text {rad }}$} \\
\cline { 4 - 9 } & & & $t=10^{-7}$ & $10^{-6}$ & $10^{-5}$ & $10^{-4}$ & $10^{-3}$ & $10^{-2}$ & $10^{-1}$ \\
\hline 50,000 & 4.5 & $3.1 \times 10^{-3}$ & 3.307 & 3.026 & 2.531 & 1.979 & 1.430 & 0.842 & 0.192 \\
& & 3.1 & 3.314 & 3.039 & 2.566 & 2.079 & 1.637 & 1.087 & 0.420 \\
& & $3.1 \times 10^{3}$ & 3.465 & 3.250 & 2.788 & 2.245 & 1.729 & 1.115 & 0.407 \\
\hline 40,000 & 4.0 & $1.8 \times 10^{-3}$ & 3.186 & 2.838 & 2.274 & 1.683 & 1.083 & 0.493 & -0.140 \\
& & 1.8 & 3.227 & 2.858 & 2.333 & 1.788 & 1.297 & 0.799 & 0.180 \\
& & $1.8 \times 10^{3}$ & 3.321 & 3.059 & 2.589 & 2.086 & 1.595 & 1.007 & 0.335 \\
\hline 30,000 & 3.5 & $1.0 \times 10^{-3}$ & 2.931 & 2.437 & 1.863 & 1.336 & 0.864 & 0.377 & -0.189 \\
& & 1.0 & 3.260 & 2.894 & 2.243 & 1.565 & 1.031 & 0.522 & -0.050 \\
& & $1.0 \times 10^{3}$ & 3.336 & 3.001 & 2.469 & 1.955 & 1.513 & 1.039 & 0.475 \\
\hline 20,000 & 2.5 & $3.0 \times 10^{-4}$ & 2.861 & 2.495 & 1.903 & 1.348 & 0.810 & 0.375 & -0.091 \\
& & $3.0 \times 10^{-1}$ & 2.952 & 2.646 & 2.283 & 1.811 & 1.357 & 0.863 & 0.275 \\
& & $3.0 \times 10^{2}$ & 3.196 & 2.857 & 2.414 & 1.948 & 1.505 & 1.031 & 0.476 \\
\hline 15,000 & 2.0 & $1.3 \times 10^{-4}$ & 2.499 & 2.231 & 1.900 & 1.377 & 0.891 & 0.430 & -0.125 \\
& & $1.3 \times 10^{-1}$ & 2.841 & 2.411 & 1.909 & 1.442 & 0.987 & 0.544 & 0.026 \\
& $1.3 \times 10^{2}$ & 3.254 & 2.938 & 2.599 & 2.283 & 1.831 & 1.279 & 0.660 \\
\hline 10,000 & 1.5 & $3.2 \times 10^{-5}$ & 2.560 & 2.300 & 1.981 & 1.633 & 1.163 & 0.603 & -0.010 \\
& & $3.2 \times 10^{-2}$ & 2.919 & 2.623 & 2.304 & 1.902 & 1.391 & 0.808 & 0.185 \\
& $3.2 \times 10^{2}$ & 3.099 & 2.757 & 2.379 & 1.942 & 1.417 & 0.835 & 0.214 \\
\hline \hline
\end{tabular}

Table 2: The parameters of the force multiplier for $O B$ supergiants

\begin{tabular}{|cc|ccc|ccc|}
\hline \hline & & \multicolumn{3}{|c|}{ this work } & \multicolumn{3}{c|}{ Abbott (1982) } \\
\cline { 3 - 8 }$T_{\text {eff }}$ & $\log g$ & $\alpha$ & $\delta$ & $k$ & $\alpha$ & $\delta$ \\
\hline 50,000 & 4.5 & 0.917 & 0.510 & 0.040 & 0.240 & 0.561 & 0.083 \\
40,000 & 4.0 & 0.483 & 0.526 & 0.061 & 0.205 & 0.578 & 0.098 \\
30,000 & 3.5 & 0.375 & 0.522 & 0.099 & 0.222 & 0.561 & 0.107 \\
20,000 & 2.5 & 0.709 & 0.470 & 0.089 & 0.429 & 0.510 & 0.084 \\
15,000 & 2.0 & 0.922 & 0.446 & 0.134 & 0.524 & 0.489 & 0.126 \\
10,000 & 1.5 & 0.866 & 0.454 & 0.058 & 0.494 & 0.490 & 0.047 \\
\hline \hline
\end{tabular}

